\title{
EFFETS DUS A LA PRÉSENCE D'INCLUSIONS MAGNÉTIQUES DANS LE MERCURE
}

\author{
J. PINEL, C. LEBEAU et J. ROSENBLATT
}

Institut National des Sciences Appliquées, Rennes

\begin{abstract}
Résumé. - On étudie les effets dus à la présence d'inclusions ferromagnétiques dans un supraconducteur de type I. Les susceptibilités de plusieurs échantillons différant par la taille, concentration et nature des inclusions dépendent seulement du rapport entre le champ critique thermodynamique du supraconducteur et l'aimantation permanente des grains. Le tracé des courbes d'aimantation a permis la détermination des champs de transitions pour des échantillons sphériques et cylindriques. Les valeurs expérimentales montrent des effets marqués dus à la forme et à l'aimantation de l'échantillon sur les champs de transitions. L'existence possible d'un état divisé, même en l'absence de champs externes et pour lequel l'énergie de surface entre régions $\mathbf{N}$ et $\mathbf{S}$ serait négative, est suggéré.
\end{abstract}

Abstract. - Effects due to aligned ferromagnetic inclusions in a type I superconductor are studied. The susceptibilities of a variety of samples differing in the nature, size and concentration of the inclusions depend only on the ratio between the bulk critical field of the superconductor and the permanent magnetization of the grains. Magnetization curves of spherical and cylindrical samples have given the transition fields. The experimental values strongly depend on the shape and magnetization of the sample. The possible existence of a divided state, even in the abscence of external fields, for which the wall energy between $\mathrm{N}$ and $\mathrm{S}$ regions should be negative, is suggested.

1. Introduction. - Un certain nombre d'études ont déjà été effectuées sur des supraconducteurs de type II contenant des inclusions ferromagnétiques en suspension [1]-[4]. Nous présentons, ici, des résultats expérimentaux concernant les modifications des propriétés d'un supraconducteur de type I contenant le même type d'inclusions. Nous avons étudié la transition du système en fonction de la température, par des mesures de susceptibilité en champ nul. D'autre part, nous avons tracé les courbes d'aimantation afin de déterminer les champs de transition. Les études ont porté sur des échantillons de mercure contenant des particules de Fer ou de Cobalt. Ils ont été obtenus en faisant réagir un amalgame de sodium avec une solution aqueuse de sulfate de Fer ou de Cobalt [5]. Un traitement thermique ultérieur permet d'obtenir la taille de billes désirée. Au cours de cette opération, nous pensons qu'il se forme une couche isolante [6] entre les grains et la matrice supraconductrice. Ceci permet de n'étudier que les effets propres à l'aimantation ferromagnétique des grains en excluant tout effet de proximité.

2. Echantillons et techniques expérimentales. - Les échantillons sont de forme sphérique $(\varnothing \simeq 7 \mathrm{~mm})$ et cylindrique $(\varnothing \simeq 1 \mathrm{~mm}, l \simeq 50 \mathrm{~mm})$. La concentration volumique (1 à $6 \%)$ et la taille des grains $(40$ à $300 \AA$ ) sont obtenues par des méthodes classiques de granulométrie magnétique [7]. Ces valeurs sont déduites de la mesure des champs coercitifs (qui vont de $485 \mathrm{Oe}$ à $905 \mathrm{Oe}$ ) et des aimantations rémanentes. Les échantillons aimantés à saturation conservent, en champ nul, sensiblement le tiers de l'aimantation à saturation. Les distances moyennes entre grains (quelques centaines d'Angströms) calculées d'après le pourcentage en volume sont inférieures à $\lambda$ profondeur de pénétration $\mathrm{du} \mathrm{Hg}$ pur. Notons que le rapport des résistances ( 1 100) aux températures de l'azote et de l'ambiant n'est pas fortement modifié par l'adjonction des inclusions ferromagnétiques.

Les échantillons sont placés dans une bobine de mesure dont l'inductance détermine la fréquence de résonance d'un circuit oscillateur LC. Les variations de susceptibilité $\Delta \chi$ se traduisent par un changement de la période d'oscillation, qui est mesurée au fréquencemètre avec une erreur relative inférieure à $10^{-4}$. Les courbes d'aimantation sont enregistrées à partir d'un magnétomètre basé sur un intégrateur électronique. Les aimantations peuvent également être obtenues par une méthode d'extraction. La température est mesurée à l'aide d'une résistance au carbone, placée au niveau de l'échantillon et qui pilote la fréquence d'un oscillateur [8].

3. Résultats expérimentaux. - Nous avons tracé les variations $\Delta \chi$ en fonction de la température pour toute une gamme d'échantillons différant par la nature des grains, leurs tailles, leurs concentrations et leurs aimantations rémanentes $M$. La figure 1 montre en encart un résultat typique concernant un cylindre avec 
une concentration volumique de $4 \%$ en grains de Fer d'un diamètre de $100 \AA$. En accord avec des résultats antérieurs [9], on peut remarquer que toutes les courbes de transition commencent à $T_{\mathrm{c} 0}$, température critique du $\mathrm{Hg}$ pur et ceci quelle que soit l'aimantation rémanente $M$ de l'échantillon. De plus, les courbes de transition présentent un élargissement d'autant plus grand que $M$ est élevé. On peut remarquer que pour $M=0$, la courbe de transition est pratiquement celle du mercure pur. Notons que la température de transition définie par le début de la transition reste égale à $T_{\mathrm{c} 0}$, ce qui semble confirmer l'existence de la couche isolante précédemment introduite. On peut penser attribuer ce comportement aux inhomogénéités en taille et concentration qui existent dans l'échantillon.

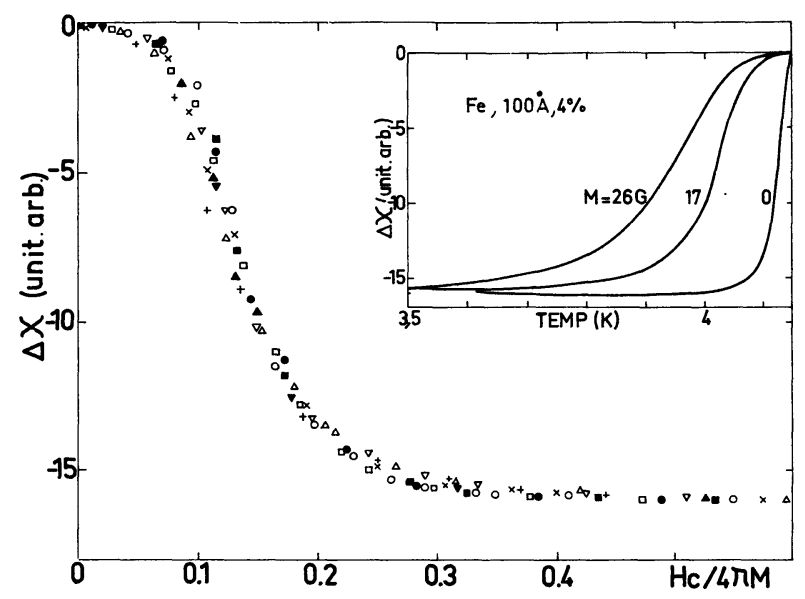

FIG. 1. - Variation de la susceptibilité en fonction de $H_{\mathrm{c}} / 4 \pi M$. Les symboles représentent respectivement le type d'inclasions, leur diamètre $(\AA)$, leur concentration en volume $(\%)$, leur aimantation moyenne (G) $\triangle: \mathrm{Fe}, 100,4,26 ; \nabla: \mathrm{Fe}, 100,4,17$; $\Delta: \mathrm{Fe}, 100,4,11 ; \nabla: \mathrm{Fe}, 100,4,5,3 ; \mathrm{O}: \mathrm{Fe}, 55,4,24$; - : $\mathrm{Fe}, 55,4,14 ; \square: \mathrm{Fe}, 320,5,28 ; \square: \mathrm{Fe}, 320,5,14$; $\times: \mathrm{Fe}, 55,2,6,13 ;+: \mathrm{Co}, 200,2,13$.

Aussi, nous avons exprimé, pour tout un ensemble d'échantillons très différents, $\Delta \chi$ en fonction d'un paramètre unique $\left(H_{\mathrm{c}} / 4 \pi M\right)$ où $H_{\mathrm{c}}$ est le champ thermodynamique critique du $\mathrm{Hg}$. La figure 1 montre que les points expérimentaux concernant une dizaine d'échantillons se placent sur une courbe unique. $\mathrm{Ce}$ résultat suggère, d'une part que les non-uniformités apparaissent sur des distances petites par rapport aux longueurs caractéristiques du supraconducteur ; ceci est d'ailleurs concevable puisqu'un volume $\lambda^{3}$ du supraconducteur contient typiquement une centaine de grains. Ce résultat indique d'autre part, que les éventuelles inhomogénéités ne jouent pas de rôle primordial dans ces expériences étant donné la faible dispersion des résultats qui permettent de tracer cette courbe.

Nous déduisons de ces mesures que le comportement $\mathrm{du}$ système dépend de ses propriétés macroscopiques à travers le paramètre unique $\left(H_{\mathrm{c}} / M\right)$. La décroissance lente de $\chi$ suggère de plus, que la coexistence de régions $\mathrm{N}$ et $\mathrm{S}$ est énergétiquement favorable pour $T \lesssim T_{\mathrm{c} 0}$.

L'existence de cet état intermédiaire est d'ailleurs confirmé par l'allure de la courbe d'aimantation d'un cylindre ; en effet, le champ appliqué $H_{\mathrm{a}}$ commence à pénétrer dans l'échantillon avant qu'il n'atteigne le champ de transition $H_{N}$. Les courbes d'aimantation ont été enregistrées par ces échantillons à différentes températures, entre $2^{\circ} \mathrm{K}$ et $4,2^{\circ} \mathrm{K} . H_{\mathrm{T}}$ est défini par l'intersection du cycle d'hystérésis ferromagnétique avec la réponse diamagnétique. Les résultats essentiels sont les suivants : pour une aimantation rémanente $M$ donnée, nous obtenons deux champs de transition différents $H^{+}(T)$ et $H^{-}(T)$ selon que $H_{\mathrm{a}}$ correspond ou non à une aimantation ferromagnétique positive. de plus, la valeur de $H_{N}$ dépend de la forme de l'échantillon comme le montre, la figure 2 . Tous ces champs de transition ne dépendent que de $M$ et pour $M_{1}=0$ nous avons $H^{+}(T) \simeq H^{-}(T) \simeq H_{\mathrm{c}}(T), \quad H_{\mathrm{c}}(T)$ étant le champ de transition du mercure pur. Nous avons montré [10] que les champs de transition de la sphère pouvaient se déduire de ceux du cylindre $H_{\mathrm{c}}^{+}$et $H_{\mathrm{c}}^{-}$. En effet, une sphère est soumise à la transition, non seulement à $H_{\mathrm{a}}$ mais aussi à un champ démagnétisant $(-4 \pi \bar{M} / 3)$ où $\bar{M}$ est une aimantation moyenne. $\mathrm{Si}$ bien que nous prévoyons : $H_{\mathrm{s}}^{ \pm}=H_{\mathrm{c}}^{ \pm} \pm 4 \pi \bar{M} / 3$. La figure 2 montre un bon accord entre les points expérimentaux pour la sphère et les valeurs calculées par la formule précédente avec $\bar{M}=0,8 \mathrm{M}$.

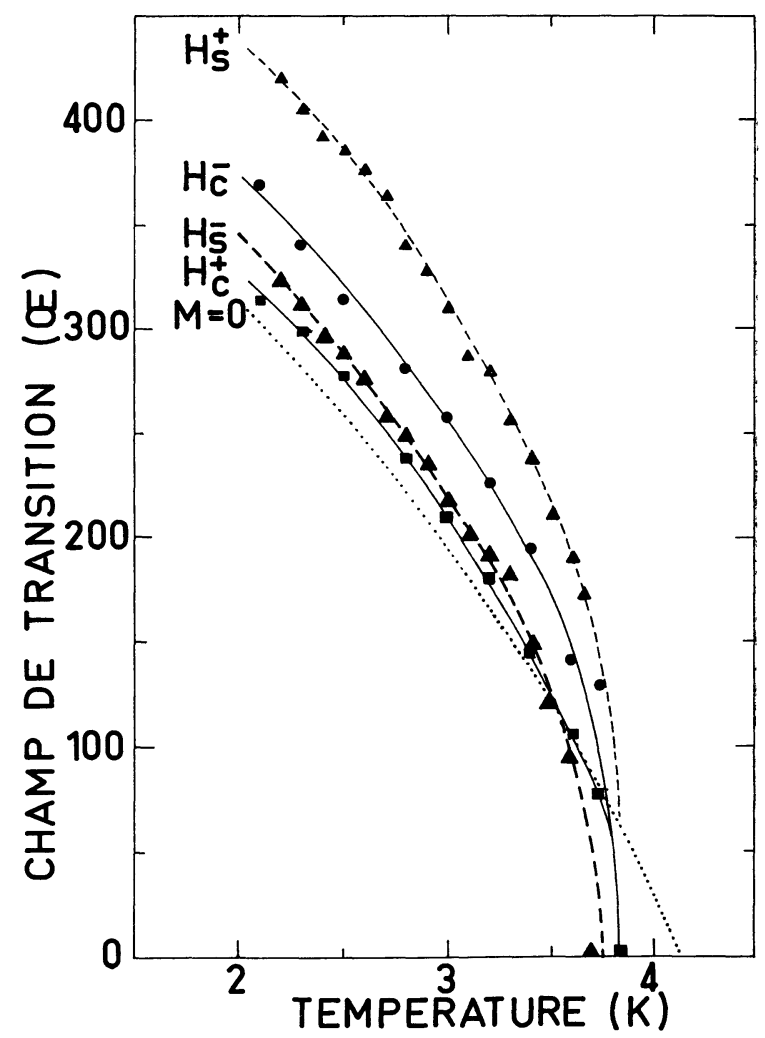

Fig. 2. - Champs de transitions $H_{\mathrm{s}}^{ \pm}$et $H_{\mathrm{c}}^{ \pm}$en fonction de la température pour des échantillons $\mathrm{Hg}-\mathrm{Fe}(100 \Lambda, 3,7 \%, 22 \mathrm{G})$ sphérique (S) et cylindrique (C). Les lignes pleines indiquent les résultats expérimentaux pour le cylindre, les triangles les points expérimentaux pour la sphère. Les lignes pointillées correspondent aux champs (S) déduits de ceux du cylindre (C) en tenant compte d'un champ démagnétisant $4 \pi \overline{M / 3}$ avec $\overline{M=0,8} \mathrm{M}$. 
4. Discussion. - L'ensemble de ces résultats expérimentaux suggère donc l'existence d'un état divisé qui existe mëme en l'absence de champ appliqué. Il serait intéressant de calculer l'énergie totale du système de façon à montrer qu'effectivement un tel état est favorable dans un certain domaine de température. On peut penser y parvenir en prenant un modèle où les billes ferromagnétiques seraient entourées de régions supraconductrices. iDans ces régions circuleraient des courants (de l'ordre 'du courant critique) ayant pour but de compenser le champ magnétique créé par chaque bille. Ce calcul est en cours. Cependant, nous avons montré [11] que la contribution dipolaire à l'énergie de surface rend celle-ci négative, ce qui est susceptible donc de favoriser un tel état intermédiaire. En effet l'énergie d'interaction entre dipôles s'écrit ( $-\mathbf{M h} / 2)$, avec $h$ le champ local s'exerçant sur chaque dipôle. Ecrivons que $h$ prend les valeurs $h_{N}$ et $h_{S}$ respectivement dans les régoins $\mathbf{N}$ et $\mathrm{S}$, et supposons que $h(x)$ varie continûment entre ces valeurs suivant une direction $x$ perpendiculaire à la paroi séparant les deux régions. L'énergie correspondant à un changement de h au point $x=0$, s'écrit simplement, en prenant une variation exponentielle du champ :

$$
E d=\frac{1}{2} \mathbf{M}\left(\mathbf{h}_{\mathrm{N}}-\mathbf{h}_{\mathrm{S}}\right) \lambda
$$

$\mathbf{M}$ ayant été pris constant dans les deux régions.
Nous pouvons estimer l'énergie de surface $E_{W}$ en supposant qu'il existe un effet Meissner total dans les. régions supraconductrices. Dans ce cas,

$$
\mathbf{h}_{\mathrm{N}}-\mathbf{h}_{\mathrm{S}}=\mathbf{B}_{\mathrm{N}}-\mathbf{B}_{\mathrm{S}}=4 \pi \mathbf{M},
$$

et alors :

$$
E_{W}=\frac{H_{\mathrm{c}}^{2} \delta}{8 \pi}-2 \pi M \lambda^{2}=\frac{H_{\mathrm{c}}^{2} \delta}{8 \pi}\left[1-\left(\frac{4 \pi M}{H_{\mathrm{c}}}\right)^{2} \frac{\lambda}{\delta}\right]
$$

le premier terme étant la contribution classique du supraconducteur à $E_{W}$ avec $\delta=1,89 \xi$ pour $\varkappa \ll 1$.

Cette expression ne prétend pas exprimer toutes les contributions d'énergie à $E_{W}$. Notamment le fait de supposer un effet Meissner total conduit certainement à une surestimation de la différence $\left(\mathbf{h}_{N}-\mathbf{h}_{S}\right)$. Toutefois cette expression permet deux remarques :

- près de $T_{\mathrm{c} 0}$, le terme $H_{\mathrm{c}}^{2} \delta \rightarrow 0$, si bien que $E_{W}$ se comporte comme $\left(-2 \pi M^{2} \lambda\right)$ qui est négatif en accord avec les constatations antérieures ;

- $\lambda / \delta$ étant une caractéristique d'un supra donné, cette expression met en évidence l'importance du rapport $\left(H_{\mathrm{c}} / 4 \pi M\right)$ en accord avec les résultats expérimentaux.

\section{Bibliographie}

[1] Alden (T. H.) et Livingston (J. D.), J. Appl. Phys., $1966,37,3551$.

[2] Brochier (D.), Cardinne (P.) et Renard (M.), $J$. Physique, 1968, 29, 953.

[3] Dobrosavljevic (L.), J. Physique, 1969, 30, 589.

[4] Hurault (J. P.) et Pincus (P.), J. of Low Temperature Phys., 1969, 1, 129.

[5] MaYeR (A.) et VoGt (E.), Z. Naturforschg., 1952, 7a, 334.

[6] HAUSER (J. J.), I heURER (H. C.) et WerThamer (N. R.), Phys. Rev., 1966, 142, 118.
[7] Luborsky (F. E.), J. Appl. Phys., 1962, 33, 1909.

[8] Lebeau (C.) et Pinel (J.), à paraître dans Journ. of Scient. Instru. E.

[9] Rosenblatt (J.), Hauser (J. J.), Valette (C.) et BuRGer (J. P.), Proceedings of L. T. 11, 1968 (University of St Andrews) Vo. II, p. 987.

[10] Lebeau (C.), Pinel (J.) et Rosenblatt (J.), J. $A . P$., 1971, 42, 323.

[11] Pinel (J.), Lebeau (C.) et Rosenblatt (J.), en voie de publication, 1971. 